\title{
Variability of Erythrocyte and Serum Lithium Levels Correlates with Therapist Treatment Adherence Efforts and Maintenance Treatment Outcome
}

\author{
Randi Taylor, B.A., Alan G. Mallinger, M.D., Ellen Frank, Ph.D., Paola Rucci, D.Stat., \\ Michael E. Thase, M.D., and David J. Kupfer, M.D.
}

This study investigated the relationship between psychotherapeutic interventions and pharmacologic measures of pharmacotherapy treatment adherence in patients with bipolar I disorder, as well as the relationship between these measures and treatment outcome. Subjects were participating in an ongoing maintenance treatment study. Audiotaped therapy sessions were rated for frequency of psychotherapeutic interventions related to pharmacotherapy treatment adherence. Pharmacologic measures of medication adherence were compared to the tape ratings as well as to treatment outcome. Variability in

KEY WORDS: Bipolar Disorder; Lithium; Patient Compliance; Psychotherapy; Treatment Outcome; Erythrocyte

It is widely recognized that many people with bipolar disorder have difficulty adhering to prescribed medication (Goodwin and Jamison 1990; Cochran and Gitlin 1988; Cochran 1984; Jamison and Akiskal 1983). Estimates of lithium noncompliance range from $20 \%$ to $66 \%$

From the Departments of Psychiatry (AGM, EF, PR, MET, DJK), Pharmacology (AGM), and Neuroscience (DJK), University of Pittsburgh School of Medicine, Department of Psychology, University of Pittsburgh (EF), Graduate School of Education, University of Pittsburgh (RT), Pittsburgh, PA, USA.

Address correspondence to: Alan G. Mallinger, M.D., Western Psychiatric Institute and Clinic, 3811 O'Hara Street, Pittsburgh, PA 15213. Tel.: (412) 624-2075; E-mail address: mallingerag@msx. upmc.edu

Received March 22, 2000; revised August 8, 2000; accepted August 25, 2000.

Presented in part at the Third International Conference on Bipolar Disorder, Pittsburgh, PA, June 17-20, 1999. log erythrocyte (RBC) lithium-a marker of probable nonadherence to the pharmacotherapy regimen-for individual patients correlated significantly with treatment adherence interventions scale ratings. This marker of nonadherence was significantly related to maintenance treatment outcome, as was variability of the serum lithium level/dose $(L / D)$ ratio; however, no relationship was found between treatment adherence interventions scale ratings and outcome. [Neuropsychopharmacology 24:192-197, 2001] (C) 2000 American College of Neuropsychopharmacology. Published by Elsevier Science Inc.

(Cochran and Gitlin 1988) in such patients. Psychotherapy is frequently employed as an adjunctive treatment strategy for bipolar disorder; however, it is not clear to what extent such intervention might enhance medication treatment adherence in this patient population. We hypothesized that therapists' efforts targeted at enhancing pharmacotherapy treatment adherence would be associated with physiologic measures of treatment adherence and clinical outcome. Therefore, we initiated a study aimed at evaluating the outcome of therapists' interventions directed toward pharmacotherapy treatment adherence, as reflected by both pharmacologic measures of treatment adherence and rates of episode recurrence.

\section{METHODS}

The Maintenance Therapies in Bipolar Disorder (MTBDMH29618, E. Frank, Principle Investigator; Frank et al. 
1999) study is an ongoing randomized trial of Interpersonal and Social Rhythm Therapy (IPSRT; Frank et al. 1994) versus an intensive clinical management (CM) control condition across both preliminary and preventative phases of treatment. Patients enter the study in the preliminary phase, during which acute treatment consisting of protocol pharmacotherapy and either IPSRT or CM is provided for their index episode of either mania or depression. Following recovery and 1 month of mood stability (defined by mean scores of $\leqslant 7$ and $\leqslant 10$ on the 17-item and 25-item versions of the Hamilton Depression Rating Scale (Hamilton 1960; Thase et al. 1992), respectively, and mean score of $\leqslant 7$ on the BechRafaelsen Mania Scale (Bech et al. 1979, for a period of 4 weeks) patients enter in the preventative phase and are again randomized to either IPSRT or CM for maintenance treatment. Both treatment approaches include a standardized psychoeducational medication adherence training component early in the preliminary phase. Written informed consent was obtained from all participants in the MTBD study apprising them of both the study description and of the possible use of the data for additional research.

At the time this study was conducted, 155 patients had been entered into the MTBD protocol. Our initial eligibility criterion for inclusion in our study was that of having been in the maintenance phase of the MTBD study for at least 1 year. This led to a pool of 63 eligible patients. The resulting sample of patients was selected utilizing the following inclusion criteria: (1) three audiotaped, ratable sessions from the first 3 months of the preventative phase; (2) five blood level determinations [which we considered the minimum to estimate standard deviation (SD) with accuracy] within 8 months of beginning the preventative phase, three of which were required to be from the first 3 months in order to correspond with the time period of the selected audiotapes; (3) being treated with lithium; and (4) remaining clinically stable during the time period when all blood level data were collected. For patients who had recurrences, all data were collected prior to the time of recurrence. Reasons for tapes not being ratable included the following: occasionally malfunctioning equipment, taping errors by the clinicians, and lost or inaudible tapes. Of the original pool of 63 eligible patients, six could not be included based on criterion 1; 17 could not be included based on criterion 2; one could not be included based on criterion 3; and eight could not be included based on criterion 4. Outcome was categorized as either recurrence or completion of 2 years maintenance without recurrence. One additional patient who dropped out of the MTBD study before a categorizable outcome could be determined was excluded.

Thirty patients were eligible for inclusion in this study. For 29 of these, we obtained the criterion number of serum lithium levels for analyses, and for 20 of the 30 participants, the criterion number of erythrocyte (RBC) lithium levels were available. (The median number of clinical blood draws per outcome group during the first 8 months of the preventative phase was eight for completers and nine for patients with at least one recurrence). Our sample was comprised of 9 males and $21 \mathrm{fe}-$ males with a study entry age range of 19-63 years (mean $\pm \mathrm{SD}=36.6 \pm 10.3$, median $=37$ ). Twenty-eight subjects were Caucasian, one was African-American and one was of Asian ethnic origin.

We developed a 12-item Likert-type scale, the Treatment Adherence Training Interventions Scale (TATIS; see Appendix), to measure therapists' efforts spent on specific types of psychotherapeutic interventions pertaining to medication treatment adherence being performed with patients in both the IPSRT and the CM treatment groups. Four students were trained to rate audiotaped IPSRT and CM sessions using this scale. The mean score of three scale ratings for each patient was compared with the following objective pharmacologic measures of medication adherence that were based on data from five time points: (1) erythrocyte lithium variability (ELV) (Harvey and Kay 1991) (calculated as SD of the log of the RBC lithium content) and coefficient of variation ( $\mathrm{CV}$, calculated as [SD/mean] $\times$ 100) of $\log$ RBC lithium content; (2) SD and CV of clinically measured serum lithium level/dose (L/D) ratios. The correlation between TATIS scores and the pharmacologic measures of medication treatment adherence was assessed using the Spearman rho test. Patients who completed 2 years of maintenance treatment without experiencing a recurrent manic or depressive episode (DSM-IV criteria) (completers) were compared with those patients who had at least one recurrence (recurrers), on both pharmacologic measures of medication treatment adherence and TATIS scores, using the Mann-Whitney U-test. TATIS scores also were compared between patients in IPSRT treatment and those receiving clinical management using the Mann-Whitney U-test. Nonparametric tests were used because of small sample sizes and because the data were for the most part not normally distributed. Demographic and clinical variables for completers and recurrers were compared using chi-square or $t$-tests. Statistical analyses were performed using SPSS 9.0 for Windows (SPSS, Inc., Chicago, IL). All tests were two-tailed.

\section{RESULTS}

No statistically significant differences were found between completers $(n=16)$ and recurrers $(n=14)$ on the following demographic and clinical variables: proportion of males/females, study entry age, duration in weeks of index manic episode, duration in weeks of in- 
dex depressive episode, baseline symptom severity, age at first mania, number of previous manic episodes, age at first depression, and number of previous depressive episodes (see Table 1). The Treatment Adherence Training Interventions Scale (TATIS) (Appendix A) mean score ratings (median $=1.33$ scored points, range $=0$ 4.33) were significantly and positively correlated with the erythrocyte lithium variability measure, ELV (median $=0.095$, range $=0.028-0.274 \log$ mmoles $\mathrm{Li} / \mathrm{L}$ RBCs; Spearman's rho $=0.50, p=.02$ ), and were marginally associated with the coefficient of variation of $\log$ RBC lithium content (median $=25 \%$, range $=6-53$, Spearman's rho $=0.39, p=.09$ ). TATIS scores were not significantly associated with either of the lithium L/D ratio-based measures (for SD of lithium L/D ratio, median $=0.09$ mmoles $/ \mathrm{L} / \mathrm{g}$, range $=0.04-0.33$ mmoles $/ \mathrm{L} /$ $\mathrm{g}$, Spearman's rho $=0.16, p=.41$; for CV of lithium L/ $\mathrm{D}$ ratio, median $=13 \%$, range $=7-44 \%$, Spearman's rho $=0.077, p=.69$ ).

For the 20 subjects for whom the criterion number of RBC lithium levels were available: ELV was significantly greater (see Figure 1), and CV of $\log$ RBC lithium content was borderline significantly greater, in the seven patients who had at least one recurrent episode (median time to recurrence from maintenance randomization $=42.4$ weeks) compared to the 13 patients who remained stable (for SD-based-ELV, median $=0.143 \mathrm{vs}$ $0.076 \log$ mmoles Li/L RBCs, respectively; Mann-Whitney $\mathrm{U}=16, p=.02$; for $\mathrm{CV}$, median $=32$ vs $17 \%$, respectively, Mann-Whitney $\mathrm{U}=21, p=.05$ ). These measures indicated that greater variability of measured RBC lithium was significantly associated with recurrent episodes.

For the 29 subjects for whom the criterion number of serum lithium levels were available: both the SD and CV (see Figure 2) of patients' lithium L/D ratios were significantly greater in the 14 patients who had at least one re- currence than in the 16 patients who remained episodefree $($ for $\mathrm{SD}$, median $=0.13 \mathrm{vs} 0.07 \mathrm{mmoles} / \mathrm{L} / \mathrm{g}$, respectively, Mann-Whitney $\mathrm{U}=47, p=.01$; for $\mathrm{CV}$, median $=$ 19 vs $9 \%$, respectively; Mann-Whitney $\mathrm{U}=32, p<.001$ ), suggesting that greater variability of serum lithium is also significantly associated with recurrence risk. No relationship was found between TATIS scores and either outcome (median $=1.17$ for completers vs 1.67 for recurrers; Mann-Whitney $U=98, p=.58$ ) or maintenance treatment assignment (median $=1$ for IPSRT vs 1.33 for $\mathrm{CM}$; Mann-Whitney $\mathrm{U}=87.5, p=.31$ ).

\section{DISCUSSION}

Results of this study provide further evidence that recurrence risk during preventative phase therapy of bipolar disorder is directly linked to nonadherence with prescribed therapy. Harvey and Kay (1991) previously examined the $\log$ RBC lithium content in relation to episode recurrence, and found ELV to be a useful parameter that was correlated with treatment outcome. They argue that logarithmic transformation of the RBC lithium level better allows for interindividual comparisons. Our observation of significantly higher ELV for those patients who suffered recurrences generally confirms Harvey and Kay's results.

The association between nonadherence and recurrence risk is particularly noteworthy because this study dealt with a relatively highly motivated and carefully selected group of patients. Moreover, by focusing on lithium responsive patients participating in a longitudinal protocol for at least one year, it is likely that highly nonadherent patients were already removed from the case mix. Thus, it appears that even a moderate degree of nonadherence has a significantly negative impact on longer term outcome.

Table 1. Subject Characteristics by Outcome Groups

\begin{tabular}{|c|c|c|}
\hline Subject Characteristics & $\begin{array}{l}\text { Recurrences } \\
\quad(n=14)\end{array}$ & $\begin{array}{l}\text { Two-year } \\
\text { completers } \\
(n=16)\end{array}$ \\
\hline $\operatorname{Sex}(M / F)^{a}$ & $4 / 10$ & $5 / 11$ \\
\hline Age $(\mathrm{yrs})^{b}$ & $35.2 \pm 8.0(37.5)$ & $38 \pm 12.0(35)$ \\
\hline Index mania duration ( $\mathrm{wks})^{b, c}$ & $10.6 \pm 9.2(8)$ & $8.1 \pm 7.3(6.5)$ \\
\hline Index depression duration (wks) ${ }^{b, c}$ & $20.4 \pm 17.5(16.0)$ & $22.3 \pm 23.1(14)$ \\
\hline Baseline symptom severity ${ }^{b, d}$ & $30.8 \pm 4.9(29.5)$ & $30.6 \pm 8.0(29.5)$ \\
\hline Age at first mania (yrs) ${ }^{b}$ & $27.6 \pm 8.0(27.5)$ & $24.8 \pm 7.4(22)$ \\
\hline Number previous manias ${ }^{b}$ & $2.6 \pm 1.9(2.5)$ & $2.8 \pm 2.3(3.0)$ \\
\hline Age at first depression $(\mathrm{yrs})^{b}$ & $21.1 \pm 6.8(19)$ & $22.1 \pm 6.6(21)$ \\
\hline Number of previous depressions $s^{b, c}$ & $4.6 \pm 5.0(4.0)$ & $5.1 \pm 6.4(3.5)$ \\
\hline
\end{tabular}

All values are reported as mean $\pm \mathrm{SD}$ (median), or counts.

a Data analyzed by chi-square.

${ }^{\mathrm{b}}$ Data analyzed by $t$-tests.

${ }^{c}$ Data were normalized by transformation as $\log (\mathrm{x})$ or $\log (\mathrm{x}+1)$

${ }^{\mathrm{d} S u m}$ of Bech-Rafaelsen mania ratings plus 25-item Hamilton depression rating. 


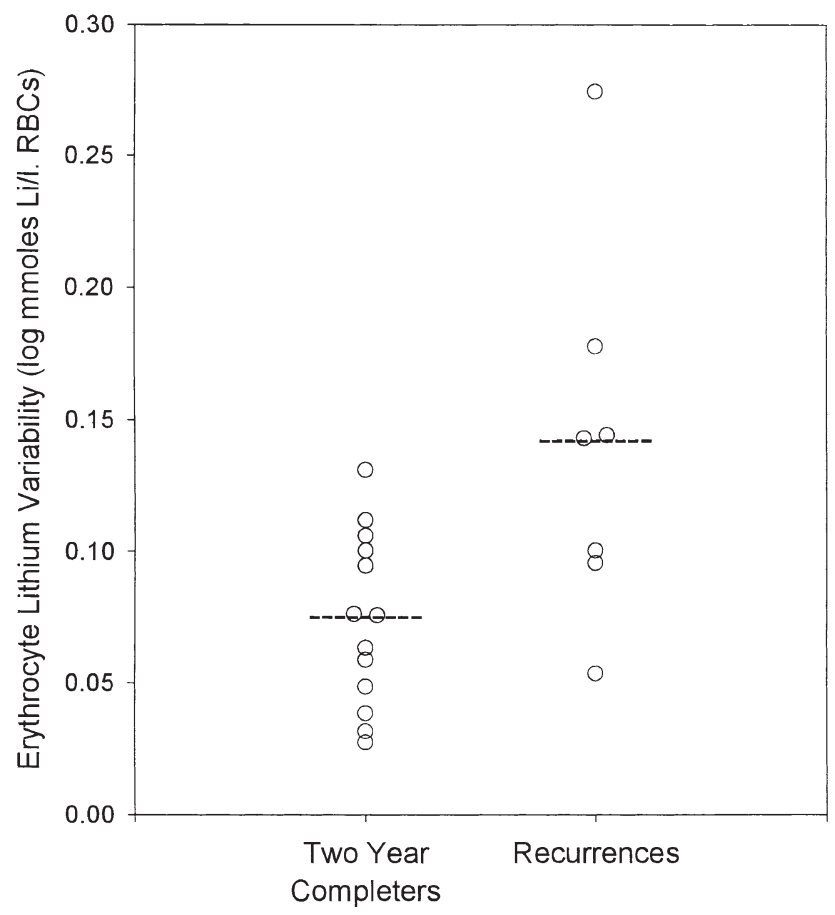

Figure 1. Plot of erythrocyte (RBC) lithium variability [calculated as standard deviation of the log of the RBC lithium content $(\log$ mmoles $\mathrm{Li} / \mathrm{L})]$ for patients completing 2 years of maintenance treatment (completers) and patients who had at least one recurrence during the preventative phase (recurrences).

There are very few existing studies specifically focusing on psychotherapeutic approaches to improving treatment adherence in patients with bipolar disorder. Cochran (1984) investigated the efficacy of a preventative treatment adherence intervention involving six weekly individual sessions focused on altering patients' cognitions and behaviors. This model was found to significantly reduce patient hospitalizations, terminations of lithium against medical advice, and the number of nonadherence-precipitated affective episodes. However, Cochran noted that objective measures of lithium nonadherence did not yield significant results, possibly due to missing or incomplete blood test data. The Treatment Adherence Training Interventions Scale was significantly and positively associated with ELV, thereby relating higher frequency of compliance-related interventions with greater variability of RBC lithium. This suggests that clinicians recognize when at least some patients are not adhering to their pharmacological treatment and attempt to intervene. Although it is possible that outcomes would have been even worse if no such interventions were attempted, our findings suggest that these interventions had limited effectiveness. Thus, more intensive approaches to enhancing medication adherence are needed. Another possible treatment approach for enhancing pharmacotherapy treatment

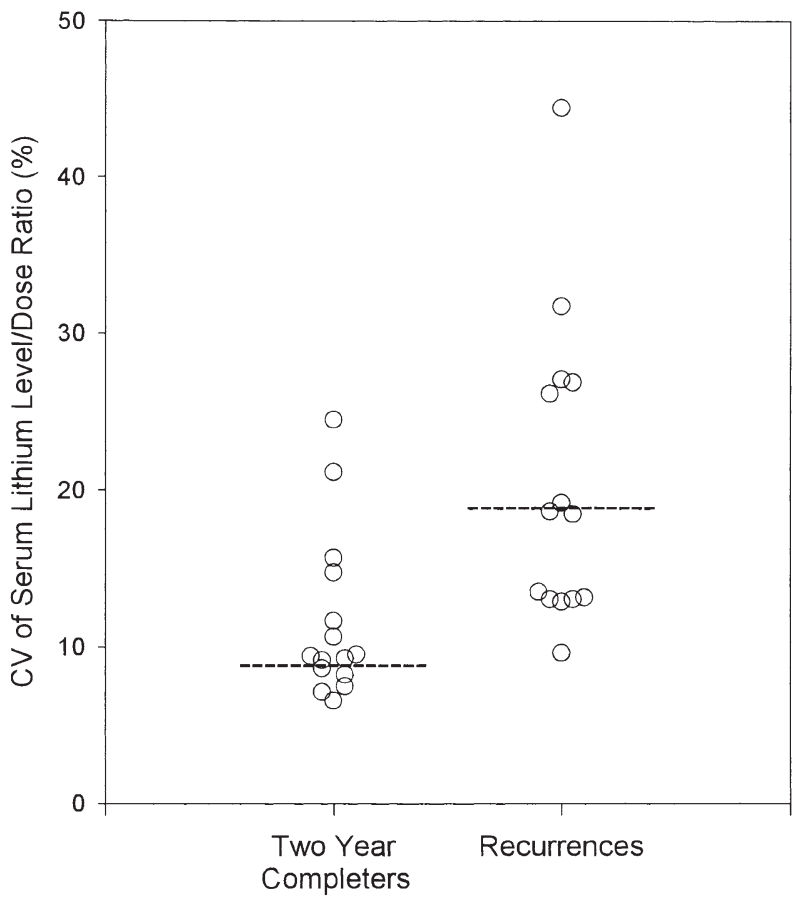

Figure 2. Plot of coefficient of variation $[\mathrm{CV}$, calculated as $(\mathrm{SD} /$ mean $) \times 100$ ] of serum lithium level/dose ratio (\%) for patients completing 2 years of maintenance treatment (completers) and patients who had at least one recurrence during the preventative phase (recurrences).

adherence in this patient population might involve some of the motivational interviewing techniques being used in the treatment of substance abuse (Miller and Rollnick 1991).

The finding that the $\mathrm{CV}$ of the lithium $\mathrm{L} / \mathrm{D}$ ratio relates to clinical outcome differs from a previously published negative observation derived from a sample partially overlapping the one presented here (Mallinger et al. 1997). We believe this reflects differences in the subject population and sample timing. The earlier investigation done by Mallinger and colleagues included early recurrences and dropouts who would not have met the inclusion criteria utilized in the present report. Moreover, only the first year of maintenance treatment was investigated in the earlier report; some patients who successfully completed this first year subsequently had recurrences during the second year. Another methodological difference is that the earlier investigation used different time points for the lithium L/D ratio observations, including some from acute treatment prior to mood stabilization. The present findings must be interpreted in the context of these differences.

Therapists' efforts spent on treatment adherence enhancement interventions were low across all patients (median $=2.33$, range $=0-4.33$, for mean score TATIS ratings, out of a possible maximum mean score of 45). This raises the general question of whether enough 
time was focused on treatment adherence during maintenance treatment. Further inquiry in this area might look at the therapist efforts spent on such interventions during the preliminary phase of treatment.

A limitation to this study was that lithium measurements were not available for all subjects from the larger study. Specifically, 17 patients from the larger study were excluded because they lacked the required number of blood samples from the time of interest. Excluding subjects because of missing blood sample data not only reduced statistical power but also adversely reduced design sensitivity because it is likely that patients who missed scheduled blood draws were also less adherent to prescribed medications. It is therefore suggested that future research in this area employ additional methods to measure treatment adherence behaviors, such as recording the number of canceled or missed appointments and using pill container devices that directly monitor medication access.

While our variability measures of both serum lithium L/D ratios and RBC lithium content were shown to be predictive of patient outcome, our investigation did not look at other important indicators of pharmacotherapy management and treatment adherence, such as the individual serum lithium levels themselves or clinical evaluation of patients' medication-taking behaviors. Hence, we are unable to make an empirical comparison between our use of lithium level variability and other currently utilized measures of pharmacotherapy treatment adherence. However, a question that we hope to address in future investigations is whether the variability measurement approach will prove to be a useful adjunct to currently used clinical methodologies, since it provides a definable method for interpreting drug level data that may otherwise be difficult to quantify, especially in cases where a patient's individual lithium levels do not demonstrate a clear pattern.

These findings, although preliminary and based on a small sample, suggest that RBC and/or serum lithium variability measures may be utilized as a benchmark means of identifying patients who are at increased risk for recurrence, presumably because of inadequate treatment adherence. Patients identified by these means might then be targeted for improved clinical interventions aimed at enhancing treatment adherence. In this way, the overall success of bipolar disorder treatment might be improved.

\section{ACKNOWLEDGMENTS}

This study was supported, in part, by National Institute of Mental Health grants MH-29618 and MH-30915.

\section{REFERENCES}

Bech P, Bolwig TG, Kramp P, Rafaelson OJ (1979): BechRafaelsen Mania Scale and the Hamilton Depression Scale. Acta Psychiatrica Scandinavica 59:420-430

Cochran SD (1984): Preventing medical noncompliance in the outpatient treatment of bipolar affective disorders. J Consult Clin Psychol 52:873-878

Cochran SD, Gitlin MJ (1988): Attitudinal correlates of lithium compliance in bipolar affective disorders. J Nervous Mental Dis 176:457-464

Frank E, Kupfer DJ, Ehlers CL, Monk TH, Cornes CL, Carter S, Frankel DR (1994): Interpersonal and social rhythm therapy for bipolar disorder, integrating interpersonal and behavioral approaches. Behav Ther 17:143-149

Frank E, Swartz H, Mallinger A, Thase M, Weaver EV, Kupfer DJ (1999): Adjunctive psychotherapy for bipolar disorder: Effects of changing treatment modality. J Abnormal Psychol 108:579-587

Goodwin FK, Jamison KR (1990): Manic Depressive Illness. New York, NY, Oxford University Press

Hamilton M (1960): A rating scale for depression. J Neurol Neurosurg Psychiat 23:56-62

Harvey NS, Kay R (1991): Compliance during lithium treatment, intra-erythrocyte lithium variability, and relapse. J Clin Psychopharmacol 11:362-367

Jamison KR, Akiskal HS (1983): Medication compliance in patients with bipolar disorder. Psychiat Clin N Am 6:175-192

Mallinger AG, Frank E, Thase ME, Dippold CS, Kupfer DJ (1997): Low rate of membrane lithium transport during treatment correlates with outcome of maintenance pharmacotherapy in bipolar disorder. Neuropsychopharmacology 16:325-332

Miller WR, Rollnick S (1991): Motivational Interviewing: Preparing People to Change Addictive Behavior. New York, NY, Guilford Press

Thase ME, Frank E, Mallinger AG, Hamer T, Kupfer, DJ (1992): Treatment of imipramine-resistant recurrent depression, III: Efficacy of monoamine oxidase inhibitors. J Clin Psychiat 53:5-10 
Appendix A. Treatment Adherence Training Interventions Scale (TATIS)

Rater\#

Patient\#

Therapist

Date

1.

To what extent did the therapist and patient explore the patient's views and/or feelings about being on lithium or other psychotropic medication?
5 extensively

Did the therapist discuss with the patient any difficulties that might have arisen in taking his/her medication as instructed?
a great deal

What portion of the session focused on helping the patient to understand his/her diagnosis of bipolar disorder, including the recurrent nature of bipolar disorder?
1
2
3
4
5
not at all
much time spent

4

5.

Did the therapist and patient discuss ways in which affective illness has negatively affected the patient's life?

$\begin{array}{lllrr}1 & 2 & 3 & 4 & 5 \\ \text { frequently } & & \text { never }\end{array}$

Did the clinician aid the patient in understanding the risks of skipping any doses of their medication?

$1 \quad 2 \quad 3 \quad 4 \quad 5$

not at all extensively

6. To what extent did the therapist and patient discuss possible interventions for lithium and/or other psychotropic medication side effects (such as coping with weight gain and excessive thirst)?
4
5
a great deal

7. Did the therapist inquire with the patient about having had any thoughts regarding not believing he/she really needs to take his/her psychotropic medication?
5 much time spent

To what extent did the clinician discuss with the patient how specific symptoms of bipolar disorder can complicate their efforts at complying with taking lithium or other psychotropic meds?
1
2
3
4
5
not at all

What portion of the session focused on helping the patient to accept his/her diagnosis of bipolar disorder?

$\begin{array}{llrrr}1 & 2 & 3 & 4 & 5 \\ \text { frequently } & & \text { never }\end{array}$

10. Did the therapist and patient discuss how the psychotropic medication treatment has changed or impacted upon the patient's life (i.e. missing of highs, impact of stabilization on relationships)? 1
4
5
great deal

Were any concerns regarding the patient's blood level (plasma or serum) discussed during the session?
2
frequently
not at all

12. Did the therapist inquire with the patient about whether or not he/she was taking his or her prescribed medication regularly as instructed? 